\title{
PRESSURE EQUILIBRIUM BETWEEN THE LOCAL INTERSTELLAR CLOUDS AND THE LOCAL HOT BUBBLE
}

\author{
S. L. Snowden ${ }^{1}$, M. Chiao ${ }^{1}$, M. R. Collier ${ }^{1}$, F. S. Porter ${ }^{1}$, N. E. Thomas ${ }^{1}$, T. Cravens ${ }^{2}$, I. P. Robertson ${ }^{2}$, \\ M. Galeazzi ${ }^{3}$, Y. Uprety ${ }^{3}$, E. Ursino ${ }^{3}$, D. Koutroumpa ${ }^{4}$, K. D. Kuntz ${ }^{5}$, R. Lallement ${ }^{6}$, L. Puspitarini ${ }^{6}$,

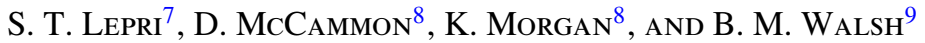 \\ ${ }^{1}$ NASA/Goddard Space Flight Center, Greenbelt, MD 20771, USA; steven.1.snowden@ nasa.gov \\ ${ }^{2}$ Department of Physics and Astronomy, University of Kansas, 1251 Wescoe Hall Drive, Lawrence, KS 66045, USA \\ ${ }^{3}$ Department of Physics, University of Miami, 1320 Campo Sano Drive, Coral Gables, FL 33146, USA \\ ${ }^{4}$ Université Versailles St-Quentin, Sorbonne Universités, UPMC Univ. Paris 06, CNRS/INSU, LATMOS-IPSL, \\ 11 Boulevard d'Alembert, F-78280 Guyancourt, France \\ 5 The Henry A. Rowland Department of Physics and Astronomy, The Johns Hopkins University, Baltimore, MD 21218, USA \\ ${ }^{6}$ GEPI, Observatoire de Paris, CNRS UMR8111, Université Paris Diderot, 5 Place Jules Janssen, F-92190 Meudon, France \\ ${ }^{7}$ University of Michigan, 2455 Hayward Street, Ann Arbor, MI 48109, USA \\ ${ }^{8}$ Department of Physics, University of Wisconsin, 1150 University Avenue, Madison, WI 53706, USA \\ ${ }^{9}$ Space Sciences Laboratory, 7 Gauss Way, Berkeley, CA 94720, USA \\ Received 2014 May 9; accepted 2014 May 28; published 2014 July 28
}

\begin{abstract}
Three recent results related to the heliosphere and the local interstellar medium (ISM) have provided an improved insight into the distribution and conditions of material in the solar neighborhood. These are the measurement of the magnetic field outside of the heliosphere by Voyager 1, the improved mapping of the three-dimensional structure of neutral material surrounding the Local Cavity using extensive ISM absorption line and reddening data, and a sounding rocket flight which observed the heliospheric helium focusing cone in X-rays and provided a robust estimate of the contribution of solar wind charge exchange emission to the ROSAT All-Sky Survey 1/4 keV band data. Combining these disparate results, we show that the thermal pressure of the plasma in the Local Hot Bubble (LHB) is $P / k=10,700 \mathrm{~cm}^{-3} \mathrm{~K}$. If the LHB is relatively free of a global magnetic field, it can easily be in pressure (thermal plus magnetic field) equilibrium with the local interstellar clouds, eliminating a long-standing discrepancy in models of the local ISM.
\end{abstract}

Key words: ISM: bubbles - ISM: clouds - magnetic fields - solar neighborhood - X-rays: diffuse background X-rays: ISM

\section{INTRODUCTION}

The observed surface brightness of the soft X-ray diffuse background (SXRB) at $1 / 4 \mathrm{keV}$ is closely linked to the local structure of the interstellar medium (ISM). There are shadowing features demonstrating the clear existence of foreground and background emission regions relative to many ISM clouds within a few hundred parsecs of the Sun (e.g., Snowden et al. 2000). The existence of a non-zero X-ray flux in the Galactic plane, where nearby $\mathrm{HI}$ column densities of a few times $10^{20} \mathrm{~cm}^{-2}$ are sufficient to be optically thick, further requires a local origin for that flux. Combining the knowledge of a local deficiency of neutral hydrogen (e.g., Knapp 1975), and the use of reddening and optical absorption line measurements to map the geometry of that deficiency (e.g., Frisch \& York 1983; Paresce 1984), led to the identification of the Local Cavity (LC) in the $\mathrm{H}$ i of the Galactic disk near the Sun. The requirement for a local source of X-ray emission coupled with the existence of the LC led to the concept of the Local Hot Bubble (LHB), where the LC contains an X-ray emitting thermal plasma with a temperature of $T \sim 10^{6} \mathrm{~K}$. The LHB is confined within the LC but does not completely fill it, extends from $\sim 50-200 \mathrm{pc}$ from the Sun, and is preferentially elongated toward high Galactic latitudes (see Sanders et al. 1977; Cox \& Snowden 1986; Snowden et al. 1990). Reviews of the $1 / 4 \mathrm{keV}$ SXRB can be found in McCammon \& Sanders (1990) and Snowden (2002).

One well-known problem with the existence of the LHB was that the required thermal pressure of the plasma was much larger than the thermal pressure of the local interstellar clouds (LICs) surrounding the Sun (e.g., Jenkins 2009). While magnetic field pressure within the LICs could account for some of the difference, the required field strength for equilibrium was unreasonably large.

An additional complication to the LHB picture came with the identification of solar wind charge exchange (SWCX) emission as a possible source for a significant flux of diffuse X-rays at $1 / 4 \mathrm{keV}$. SWCX X-ray emission occurs when highly ionized metals in the solar wind interact with neutral material gaining an electron in a highly excited state, which then decays emitting a photon (or photons) with a characteristic energy of the ion. First identified in ROSAT observations of comets (Lisse et al. 1996; Cravens 1997), Cox (1998) and Freyberg (1998) suggested that SWCX could be the source for the non-cosmic background enhancements observed in the ROSAT All-Sky Survey (RASS; Snowden et al. 1995). Cravens et al. (2001) confirmed this by showing the strong correlation between solar wind flux and the background enhancement count rate.

The question was then: how much of the $1 / 4 \mathrm{keV}$ SXRB originates in the LHB and how much originates as SWCX with interstellar neutrals flowing through the solar system (e.g., Lallement 2004; Welsh \& Shelton 2009) or with exospheric material in Earth's magnetosheath (e.g., Robertson \& Cravens 2003)? While the temporally varying SWCX component, with timescales from a few hours to a few days, was modeled and removed from the RASS, any longer-term variations (such as those arising as distributed emission through the heliosphere where the variation is smoothed out) could not, and so remain as an uncertain zero-level offset in the RASS maps. It was 
that unknown offset that might possibly have provided all of the observed local emission and thus obviated the need for the LHB.

\section{NEW TOOLS FOR THE LICs AND LHB}

Three recent advances have shed considerable light on the relationship between the LICs and the LHB: (1) the Diffuse $\mathrm{X}$-rays from the Local galaxy (DXL) sounding rocket experiment (Galeazzi et al. 2011) produced a measurement of the amount of SWCX emission from the heliosphere that contributes to the RASS 1/4 keV map (Galeazzi et al. 2014, hereafter G14); (2) the three-dimensional mapping of neutral material in the local ISM using absorption line and reddening data has greatly improved our knowledge of its distribution in the nearest $\sim 500 \mathrm{pc}$ from the Sun (Lallement et al. 2014; Puspitarini et al. 2014); and (3) the penetration of the Voyager 1 spacecraft beyond the heliosphere has allowed a more reliable in situ measurement of the interstellar magnetic field within the LICs (Burlaga \& Ness 2014).

\subsection{X-Ray Data}

G14 provides an estimate for the heliospheric and magnetospheric SWCX contribution to the RASS (Snowden et al. 1997) $1 / 4 \mathrm{keV}$ band ( $R 12$ band) by fitting the observed count rate along the DXL scan path, the RASS count rate for the scan path, and the model SWCX emission from the heliosphere calculated for the sounding rocket and RASS observation geometries and solar wind conditions. The fractional contribution of SWCX emission to the RASS data along the DXL scan path is $0.40 \pm 0.07$ (combining systematic and statistical errors in quadrature), typically $140 \mathrm{RU}$ (ROSAT units, $10^{-6}$ counts $\mathrm{s}^{-1} \operatorname{arcmin}^{-2}$ ). Using the HEASARC X-ray Background Tool, ${ }^{10}$ a RASS spectrum was extracted for a 4.5 radius region centered on $l \sim 144^{\circ}, b \sim 0^{\circ}$, the direction of minimum surface brightness along the DXL scan path. The $R 12$ count rate for the extraction is $339 \pm 2 \mathrm{RU}$. (Note that $0.40 \pm 0.07$ of the extracted count rate is $136 \pm 24 \mathrm{RU}$, consistent with the typical SWCX flux value of 140 RU stated in G14.) The spectrum was fit (using $\mathrm{Xspec}^{11}$ ) with a simple three-component model for the diffuse X-ray background consisting of (1) an unabsorbed thermal emission component representing the sum of emission from SWCX and the LHB, (2) an absorbed thermal component representing emission from more distant regions of the Milky Way, and (3) an absorbed power-law component representing the extragalactic background. The fitted parameters for the unabsorbed component were a temperature of $k T=0.102 \pm 0.001 \mathrm{keV}\left(T=(1.18 \pm 0.01) \times 10^{6} \mathrm{~K}\right)$, and an Xspec normalization of $5.37_{-0.10}^{+0.05} \times 10^{-7}$ for an emission measure $n_{e} n_{\mathrm{H}} L=2.59_{-0.05}^{+0.02} \times 10^{-3} \mathrm{~cm}^{-6} \mathrm{pc}$, where $L$ is the path length through the emission in parsecs and $n_{e}$ and $n_{\mathrm{H}}$ are the electron and proton densities in the plasma, respectively (assumed to be constant along the path length). Current APEC (Smith et al. 2001) thermal emission models with Anders \& Grevesse (1989) abundances were used for the fit. The fitted absorption column density was $\sim 1.3 \times 10^{21} \mathrm{~cm}^{-2}$ so the two absorbed spectral components have no contribution to the $R 12$ band. While there is some dependence on the choice of thermal emission model, the APEC model was the most conservative as

\footnotetext{
10 http://heasarc.gsfc.nasa.gov/cgi-bin/Tools/xraybg/xraybg.pl

$11 \mathrm{Xspec}$ is the HEASARC spectral fitting package: http://heasarc.gsfc.nasa. gov/docs/xanadu/xspec/index.html
}

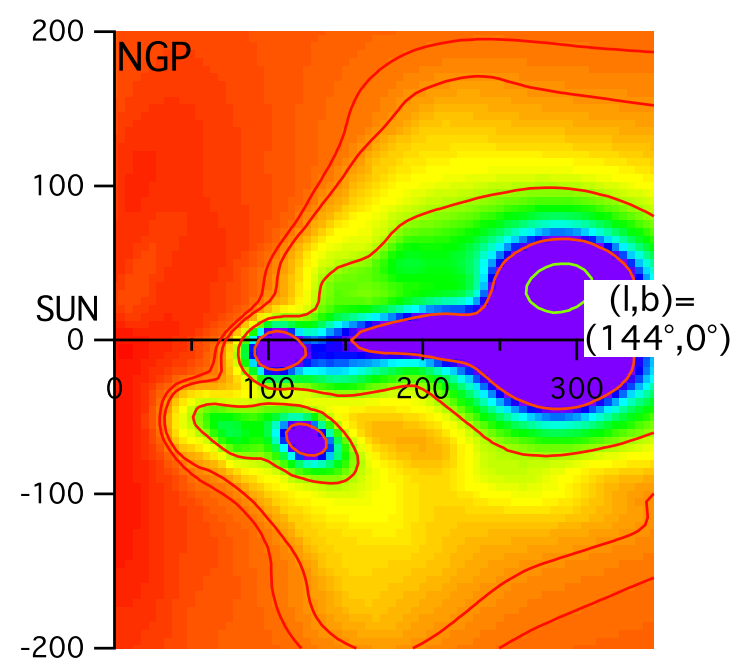

Figure 1. Distribution of the neutral ISM in the direction of $l \sim 144^{\circ}, b \sim 0^{\circ}$ from the analysis of Lallement et al. (2014) where purple indicates the denser material of the LC wall and the distances are in parsecs. The inversion mapping is based on reddening and absorption line data, and is currently limited by the number of sample stars to a resolution of $\sim 15 \mathrm{pc}$.

it led to the highest normalization and thermal pressure. There is also a dependence on assumed elemental abundances, however, using the Savage \& Sembach (1996) warm cloud abundances led to a thermal pressure only $\sim 15 \%$ higher and a required LIC magnetic field pressure for equilibrium only $\sim 10 \%$ higher than that derived below.

Subtracting the SWCX contribution from the RASS yields $203 \pm 24$ RU for the LHB emission. Scaling the fitted Xspec emission measure by $203 / 339$ to represent just the LHB emission, and assuming that the LHB and SWCX spectra are similar (demonstrated during the processing of the RASS data; Snowden et al. 1995), yields an inferred emission measure for the LHB component of $n_{e} n_{p} L=(1.55 \pm 0.18) \times 10^{-3} \mathrm{~cm}^{-6} \mathrm{pc}$.

\subsection{Interstellar Thermal Pressure}

From Lallement et al. (2014), the distance to the nearest wall of $\mathrm{HI}_{\mathrm{I}}$ in the direction of $l \sim 144^{\circ}, b \sim 0^{\circ}$ is $\sim 90 \pm 15 \mathrm{pc}$ (see Figure 1). However, part of that path length is filled by the LICs so we subtract an estimate of 5 pc (Frisch et al. 2011) leaving a plasma path length of $85 \pm 15 \mathrm{pc}$ (assuming that the error in the path length in the LICs is much smaller than the uncertainty in the distance to the edge of the LC). Again, assuming that $n_{e}$ and $n_{\mathrm{H}}$ are constant along the line of sight and using Xspec's assumption that $n_{e} n_{\mathrm{H}}=n_{e}^{2} / 1.2$, we have

$$
n_{e}=(4.68 \pm 0.47) \times 10^{-3} \mathrm{~cm}^{-3} .
$$

The thermal pressure of the plasma is then

$$
P_{T} / k=n T=10600 \pm 1070 \mathrm{~cm}^{-3} \mathrm{~K},
$$

where $n$ is the total particle density $\left(n \sim 1.92 n_{e}\right)$. This is $\sim 60 \%$ the LHB thermal pressure estimated by Galeazzi et al. (2007), which well represents previous measurements that did not include the effect of SWCX emission. (Note that the evolution of thermal models can affect the pressure scaling, as well as the accuracy of assumed path length through the emitting plasma.) On the other hand, the value of $P_{T} / k=10,200 \mathrm{~cm}^{-3} \mathrm{~K}$ derived in Puspitarini et al. (2014) is in quite good agreement. From Frisch et al. (2011), the LIC has a thermal pressure of $P_{T} / k=$ $3200_{-1100}^{+2000} \mathrm{~cm}^{-3} \mathrm{~K}$, which implies a thermal pressure difference between the LICs and the LHB of $\Delta\left(P_{T} / k\right) \sim 7400_{-2270}^{+1530} \mathrm{~cm}^{-3} \mathrm{~K}$. 


\subsection{Interstellar Magnetic Field Pressure}

Assuming that the LHB plasma has been swept relatively free of magnetic fields, the magnetic pressure within the LICs may compensate for the difference in thermal pressures. (Also assuming that other contributors to the total interstellar pressure either similarly affect both the LICs and the LHB or are negligible.) The magnetic field pressure is $P_{\mathrm{B}} / k=$ $288 B^{2} \mathrm{~cm}^{-3} \mathrm{~K}$ where $B$ is in $\mu \mathrm{G}$. The required LIC magnetic field for pressure equilibrium is then

$$
B=\sqrt{\Delta\left(P_{T} / k\right) / 288}=5.1_{-0.9}^{+0.5} \mu \mathrm{G}
$$

From Burlaga \& Ness (2014), the interstellar magnetic field in the LIC ranges from $3.8 \mu \mathrm{G}$ to $5.9 \mu \mathrm{G}$ with an average of $4.9 \pm 0.5 \mu \mathrm{G}$.

\section{DISCUSSION}

Assuming that the magnetic field measured by Voyager 1 is representative of the LICs, the agreement between the thermal plus magnetic field pressure of the LICs and the thermal pressure of the LHB plasma alleviates a long-standing inconsistency in the LHB model leading to a self-consistent picture of the local ISM. The pressure equilibrium explains why there has been no evidence for either the expansion or contraction of the LICs (Frisch et al. 2011), and is consistent with the picture of a rather sedate and aged bubble of hot plasma. (Note, however, that Gry \& Jenkins 2014 have a new interpretation that the LICs are actually one monolithic structure which is compressing in the ram and anti-ram directions and expanding perpendicularly to the flow.), The results of G14 indicate that $\sim 40 \%$ of the minimum observed $1 / 4 \mathrm{keV}$ flux in the Galactic plane originates as SWCX emission within the heliosphere and magnetosheath but still requires X-ray emitting plasma within the LC, even at low Galactic latitudes (the required LC/LHB plasma at higher latitudes is relatively unchanged). In short, contrary to rumors of its demise (e.g., Welsh \& Shelton 2009), the LHB still lives.

A recent paper by Smith et al. (2014) came to a similar conclusion about the continued existence of the LHB using a spectral analysis of Diffuse X-ray Spectrometer (DXS; Sanders et al. 2001) and Cosmic Hot Interstellar Plasma Spectrometer (Hurwitz et al. 2005) data. Using their own model for the SWCX spectrum plus a thermal emission component they fit the high spectral resolution DXS $1 / 4 \mathrm{keV}$ band data deriving the result that $26 \% \pm 4 \%$ of the observed emission was from the LHB (the DXS data were from near the Galactic plane). While the fraction of the observed emission from the LHB is less than half of the amount found by G14, the Smith et al. (2014) results suggest a thermal pressure considerably closer to that of the LICs, and thus less reliant on a differential in magnetic field strength for pressure equilibrium.

The local ISM is clearly more complicated than simply filling the LC with a hot plasma. For instance, there are directions where neither neutral material nor X-ray emitting plasma are observed, such as the Region of Bizarre Emptiness (RBE; Cox 1998) although a large filling factor of $\mathrm{H}$ II region-like-gas, such as observed in the direction of $\beta$ CMa (Gry et al. 1985) could be responsible.

While we report here the characterization of the LHB, SWCX, LIC, and thermal and magnetic field pressures based on the data in one direction, these results can reasonably be extended to the entire LHB. As mentioned above, the LHB thermal pressure that is derived here is in very good agreement with the thermal pressure derived in Puspitarini et al. (2014), which considered the LHB's extent in the LC. With their scaling, the plasma required for the LHB emission fits inside their three-dimensional mapping of the LC.

We are grateful to Ed Jenkins for interesting and helpful insights into these results. This work has been supported in part by NASA's award \#NNX11AF04G.

\section{REFERENCES}

Anders, E., \& Grevesse, N. 1989, GeCoA, 53, 197

Burlaga, L. F., \& Ness, N. F. 2014, ApJ, 784, 146

Cox, D. P. 1998, in IAU Colloq. 166, The Local Bubble and Beyond, ed. D. Breitschwerdt, M. J. Freyberg, \& J. Truemper (Lecture Notes in Physics, Vol. 506; Berlin: Springer), 121

Cox, D. P., \& Snowden, S. L. 1986, AdSpR, 6, 97

Cravens, T. E. 1997, GeoRL, 24, 105

Cravens, T. E., Robertson, I. P., \& Snowden, S. L. 2001, JGR, 106, 24883

Freyberg, M. J. 1998, in IAU Colloq. 166, The Local Bubble and Beyond, ed. D. Breitschwerdt, M. J. Freyberg, \& J. Truemper (Lecture Notes in Physics, Vol. 506; Berlin: Springer), 113

Frisch, P. C., Redfield, S., \& Slavin, J. D. 2011, ARA\&A, 49, 237

Frisch, P. C., \& York, D. G. 1983, ApJL, 271, L59

Galeazzi, M., Gupta, A., Covey, K., \& Ursino, E. 2007, ApJ, 658, 1081

Galeazzi, M., Chiao, M., Collier, M. R., et al. 2011, ExA, 32, 83

Galeazzi, M., Chiao, M., Collier, M. R., et al. 2014, Natur, in press

Gry, C., \& Jenkins, E. B. 2014, arXiv:1404.0326

Gry, C., York, D. G., \& Vidal-Madjar, A. 1985, ApJ, 296, 593

Hurwitz, M., Sasseen, T. P., \& Sirk, M. M. 2005, ApJ, 623, 911

Jenkins, E. B. 2009, SSRv, 143, 205

Knapp, G. R. 1975, AJ, 80, 111

Lallement, R. 2004, A\&A, 422, 391

Lallement, R., Vergely, J.-L., Valette, B., et al. 2014, A\&A, 561, A91

Lisse, C. M., Dennerl, K., Englhauser, J., et al. 1996, Sci, 274, 205

McCammon, D., \& Sanders, W. T. 1990, ARA\&A, 28, 657

Paresce, F. 1984, AJ, 89, 1022

Puspitarini, L., Lallement, R., Vergely, J. L., \& Snowden, S. L. 2014, A\&A, 566, A13

Robertson, I. P., \& Cravens, T. E. 2003, GeoRL, 30, 1439

Sanders, W. T., Edgar, R. J., Kraushaar, W. L., McCammon, D., \& Morgenthaler, J. P. 2001, ApJ, 554, 694

Sanders, W. T., Kraushaar, W. L., Nousek, J. A., \& Fried, P. M. 1977, ApJL, 217, L87

Savage, B. D., \& Sembach, K. R. 1996, ApJ, 470, 893

Smith, R. K., Brickhouse, N. S., Liedahl, D. A., \& Raymond, J. C. 2001, ApJL, 556, L91

Smith, R. K., Foster, A. R., Edgar, R. J., \& Brickhouse, N. S. 2014, ApJ, 787,77

Snowden, S. L. 2002, in The Century of Space Science, Vol. I, ed. J. A. Bleeker, J. Geiss, \& M. C. E. Huber (Dordrecht: Kluwer), 581

Snowden, S. L., Cox, D. P., McCammon, D., \& Sanders, W. T. 1990, ApJ, 354,211

Snowden, S. L., Egger, R., Freyberg, M. J., et al. 1997, ApJ, 485, 125

Snowden, S. L., Freyberg, M. J., Kuntz, K. D., \& Sanders, W. T. 2000, ApJS, 128,171

Snowden, S. L., Freyberg, M. J., Plucinsky, P. P., et al. 1995, ApJ, 454, 643

Welsh, B. Y., \& Shelton, R. L. 2009, Ap\&SS, 323, 1 